\title{
On the mathematical theory of classical fields and general relativity
}

Sergiu Klainerman*

From the perspective of an analyst, like myself, the General Theory of Relativity provides an extrordinary rich and vastly virgin territory. It is the aim of my lecture to provide, first, an account of those aspects of the theory which attract me most and second a perspective of what has been accomplished so far in that respect. In trying to state our main objectives it helps to view General Relativity in the broader context of Classical Field Theory. As we know today, among all classical field theories, only the Maxwell equations and the Einstein field equations are known to have direct physical content. Others, like the Yang-Mills equations, are believed to become relevant only after they suffer the painful and still mysterious, process of quantization. In view of this fact one can describe the basic goals of the mathematical theory of classical fields as follows:

(1) Investigate the mathematical consequences of the Einstein Field Equations in so far as they describe the physical world.

(2) Investigate the mathematical properties of the other nonlinear field theories

(a) In view of the fact that the Einstein-field equations are exceedingly complicated some of the other field theories provide a simplified testing ground for new ideas. From this point of view the final goal remains the understanding of the Einstein field equations but we hope to get important insights from the simpler nonlinear field theories. To this one might add our belief that any new icleas which lead to significant progress made on problems of nonlinear classical fields will prove useful in other areas of Mathematical Physics where nonlinear P.D.E's, in particular hyperbolic, are at the heart of the subject.

(b) Investigate the mathematical properties of nonlinear field theories in view of their possible relevance to quantum field theory.

This final goal is most vague. One may attempt to render it slightly more meaningful by pointing out the importance of uncovering the fundamental properties of classical mechanical systems as carried out by mathematicians such as Euler, Lagrange, Hamilton, Poincare and others, to the very formulation of Quantum Mechanics.

Since according to the above scheme, General Relativity and the Einstein field equations play the dominant role in the mathematical theory of classical fields, I will concentrate my attention to it and refer to other field theories from the perspective of 2a. The mathematical structure of the Einstein-Vacuum equations, or shortly E-V, is already sufficiently complicated, I will thus restrict my attention to them.

Clearly, the aim of the mathematical theory of General Relativity is to understand the main features concerning the behaviour of general solution to the Einstein Field equations. Since general solutions can be naturaly parametrized by initial data sets one can reformulate our goal as being that of studying the main features of the evolution of general initial data sets. Now this goal is too broad since without an appropriate asymptotic restriction the evolution of an arbitrary initial data set can be very wild. A reasonable physical restriction is to consider initial data sets which look flat outside a

* Supported by the N.S.F. grant DMS-9103613 
sufficiently large compact set of $\mathcal{H}$. The evolution of such initial data sets correspond to "isolated physical systems." These systems are particularly important in G.R. since, it is only for such systems that we can define the physical notions of mass, linear and angular momentum.

We thus redefine our central theme as being the study of the main features regarding the evolution of general classes of asymptotically flat initial data sets. From the perspective of $(2 \mathrm{a})$ we can broaden this to include all classical field theories. In this respect I will restrict myself to a discussion of field theories in Minkowski space-time.

Having thus defined our object of study I can turn my attention to what is considered to be the most fundamental mathematical question concerning the differential equations of General Relativity and the other classical Field theories. This is to understand when and how, solutions to a classical, nonlinear, field theory can break-down.

The break-down phenomenon can occur despite the existence of the basic conservation laws, in particular the energy, or total mass, which is positive. To understand what this means consider the comparable situation in one dimension, namely systems of ordinary differential equations which arise as the Euler-Lagrange equations of a Lagrangean with positive energy. To be more precise consider the example of the differential equation,

$$
-\ddot{a}+V^{\prime}(x)=0
$$

subject to the initial conditions at $t=0$,

$$
x(0)=x_{0}, \dot{x}(0)=x_{1} .
$$

The total energy of the system is given by the expression $\frac{1}{2}|\dot{x}|^{2}-V(x)$, where $\frac{1}{2}|\dot{x}|^{2}$ is the kinetic energy and $-V$ the potential energy. For any reasonable physical system $-V \geq 0$. Since the total energy is conserved we immediately conclude that, for any initial conditions, the corresponding solutions exist for all time. There can be no finite time blow-up of the solutions.

The situation is very different for classical field theories. Though we have a positive energy momentum tensor which leads, in Minkowski space-time, to well defined conserved quantities, we cannot infer in general that the solutions, starting with perfectly smooth inital conditions, remain so for all time. Take as an example the field theory closest to our one dimensional example, namely the scalar wave equation in Minkowski space-time $\mathbf{M}^{n+1}$,

$$
\square \phi+V^{\prime}(\phi)=0,
$$

subject to the initial conditions at $t=0$,

$$
\phi(0)=f_{0}, \quad \partial_{t} \phi(0)=f_{1}
$$

Assume that $f_{0}, f_{1}$ are as regular as we want, say $f_{0}, f_{1} \in \mathcal{C}_{0}^{\infty}\left(\Re^{n}\right)$. Assume also that $-V \geq 0$ so that the energy-momentum tensor satisfies the required positivity condition.

The total energy ${ }^{1}$ at time $t$ has the form,

$$
E(t)=\int_{\Re^{n}}\left(\frac{1}{2}\left(\left(\partial_{t} \phi\right)^{2}+\left(\partial_{1} \phi\right)^{2} \cdots+\left(\partial_{n} \phi\right)^{2}\right)-V(\phi)\right) d x
$$

1 which is the conserved quantity corresponding to the Killing vectorfield $T_{0}=\partial_{t}$ 
One can easily check directly that $\frac{d}{d t} E(t)=0$ and hence, if the initial data at time $t=0$ are such that $E(0)$ is bounded, we infer that $E(t)$ is bounded for all time. Yet, unlike in the previous example we cannot conclude that the solutions remain smooth at all later times. It is easy to see that as long as $\phi$ remains bounded its time evolution preserves the regularity of the initial conditions in the $L^{2}$ norm. The problem, thus, is to show that $\phi$ stays bounded. In space dimension $n=1$ we can conclude, from the boundedness of $E(t)$ and a simple form of the Sobolev inequalities, that $\phi$ is point-wise bounded. Indced, since $-V \geq 0$, we infer that at any time $t$, $\int_{\Re^{n}}\left(\frac{1}{2}\left(\left(\partial_{t} \phi\right)^{2}+\left(\partial_{1} \phi\right)^{2} \cdots+\left(\partial_{n} \phi\right)^{2}\right)\right) d x \leq E(0)$. On the other hand, according to the simplest version of the basic Sobolev inequalities, the sup-norm of a function in $\mathbf{R}^{n}$ can be bounded in terms of the square integrals of the sum of all its derivatives of order ${ }^{2}$ $\left[\frac{n}{2}\right]$. Hence for $n=1$,

$$
\sup _{x}|\phi(t, x)| \leq c\left(\int_{\Re^{n} n} \frac{1}{2}\left(\left(\partial_{1} \phi\right)^{2} \cdots+\left(\partial_{n} \phi\right)^{2}\right) d x\right)^{1 / 2} \leq c E(0)^{1 / 2} .
$$

The form of the Sobolev inequality we have used above fails just a little for $n=2$. In fact we can only estimate $\left(\int|\phi(t, x)|^{p}\right)^{1 / p}$ for any $p<\infty$, this turns out nevertheless to be enough to conclude that the solutions remain smooth for all time ${ }^{3}$. For $n \geq 3$ the above form of the Sobolev inequality require a little more than $3 / 2$ derivatives in $L^{2}$ in order to estimate the sup norm of $\phi$. The boundedness of the energy provides us with a bound of only one derivative in $L^{2}$. We have thus a gap of more than $1 / 2$ derivatives. The situation gets, of course, even worse in higher dimensions. We can still, nevertheless, show that $\phi$ remains bounded provided that $V(\phi)$ does not grow too fast as $\phi \rightarrow \infty$. For simplicity consider the case of the power potential $V(\phi)=-\frac{1}{p+1}|\phi|^{p+1}$. Prescribing to the space-time variables $t=x^{0}, x^{1}, \ldots, x^{n}$ the same scale $L$, the solution $\phi$ of the equation, $\square \phi+V^{\prime}(\phi)=0$, acquires the scale $L^{-\frac{2}{p-1}}$. Therefore the total energy $E$ has the scale $L^{s}$ where $s$ is the exponent $s=n-2-\frac{4}{p-1}$.

The case when the exponent $s$ is strictly negative is called "subcritical". It is quite easy to analyze and has lead to the well-known global regularity result of Jörgens [Jö]. The case $s=0$ is called "critical " while $s>0$ is called "supercritical." In the supercritical regime we have no results, even for spherically symmetric solutions, despite the relatively large attention this problem has received. The critical case has been recently settled by the combined efforts of Struwe [Stru], Grillakis [Gr1] and more recently Shatah-Struwe [Sh-Stru].

Theorem. Consider the initial value problem $\square \phi+V^{\prime}(\phi)=0$ with initial conditions $\phi(0, x)=f_{0}(x), \partial_{t} \phi(0, x)=f_{1}(x)$ which, for simplicity, we may assume in $\mathcal{C}_{0}^{\infty}$. Assume that $n \leq 7$ and that the exponent $s \leq 0$. Then the equations admits unique smooth solutions globally in $\mathbf{M}^{n+1}$.

Despite the difficulty of the problem it is widely believed that, even in the supercritical case, the solutions to (N.W.E.) remain smooth for all time.

A more interesting field theory is provided by the equations of Wave Maps defined from Minkowski space-time $\mathbf{M}^{n+1}$ with values in a Riemannian manifold $\mathcal{N}$. Relative

$2\left[\frac{n}{2}\right]$ denotes the smallest integer strictly greater than $\frac{n}{2}$.

3 provided that $V(\phi)$ has polynomial growth in $\phi$ for large $\phi$. 
to standard coordinates $x^{\alpha}, \alpha=0, \ldots, n$ and local coordinates $y^{a}, a=1, \ldots m$ in $\mathcal{N}$ the equations take the form,

$$
\square \phi^{a}+\Gamma_{b c}^{a}(\phi) \mathbf{m}^{\mu \nu} \partial_{\mu} \phi^{b} \partial_{\nu} \phi^{c}=0
$$

where $\Gamma_{b c}^{a}$ are the Christoffel symbols of $\mathcal{N}$. Consider the initial conditions at $x^{0}=$ $t=0$,

$$
\phi(0)=f_{0}, \quad \partial_{t} \phi(0)=f_{1}
$$

with $f_{0}, f_{1}$ compactly supported smooth maps defined from $\mathbf{R}^{n} \mathbf{R}^{n}$ to $\mathcal{N}$.

Since the nonlinear terms are quadratic in the first derivatives of the map $\phi$, in order to preserve the $L^{2}$ regularity of the initial conditions, we now need to have pointwise bounds not only on $\phi$ but also its first derivatives. The total energy, in this case, has the form,

$$
E(t)=\int_{\Re^{n}}\left(\frac{1}{2}\left(\left|\partial_{t} \phi\right|^{2}+\left|\partial_{1} \phi\right|^{2} \cdots+\left|\partial_{n} \phi\right|^{2}\right)\right) d x
$$

with $\left|\partial_{\alpha} \phi\right|^{2}=h_{a b} \partial_{\alpha} \phi^{a} \partial_{\alpha} \phi^{b}, h$ the Riemannian metric of $\mathcal{N}$. The law of conservation of total energy is, as before,

$$
E(t)=E(0)
$$

This provides us with only with an $L^{2}$ bound for the derivatives of $\phi$. We therefore see that the conservation law for the total energy does not suffice to control the $L^{\infty}$ norm of the first derivatives of $\phi$ even for wave maps in $\mathbf{M}^{1+1}$. A simple remark, however, allows us to bypass the difficulty in this case (see [Sh] and also [Gu]). Indeed consider the energy momentum tensor $\mathbf{T}$. It has the form, $\mathbf{T}_{\alpha \beta}=\frac{1}{2}\left(\left\langle\phi_{, \alpha}, \phi, \beta\right\rangle-\right.$ $\frac{1}{2} \mathbf{g}_{\alpha \beta}\left(\mathbf{g}^{\mu \nu}\left\langle\phi_{, \mu} \phi_{, \nu}\right\rangle\right)$ with $<,>$ the scalar product in $\mathcal{N}$. Since we are in $1+1$ dimensions $\mathbf{T}$ has only the components $\mathbf{T}_{00}, \mathbf{T}_{01}=\mathbf{T}_{10}, \mathbf{T}_{11}$. Moreover, since $\mathbf{T}$ is trace-less in $\mathbf{M}^{1+1}$, we have $\mathbf{T}_{00}=\mathbf{T}_{11}$. Now recall that $\mathbf{T}$ verifies the divergence equation $\partial^{\beta} \mathbf{T}_{\alpha \beta}=0$. Hence,

$$
\begin{aligned}
& \partial_{t} \mathbf{T}_{00}=\partial_{x} \mathbf{T}_{01} \\
& \partial_{t} \mathbf{T}_{01}=\partial_{x} \mathbf{T}_{00}
\end{aligned}
$$

and therefore $\mathbf{T}_{00}$ is a solution of the linear wave equation in $\mathbf{M}^{1+1}, \mathbf{T}_{00}=0$. One can now easily check that $\mathbf{T}$ remains bounded for any $t>0$ provided that $\mathbf{T}_{00}, \partial_{t} \mathbf{T}_{00}$ are bounded at $t=0$. Since $\mathbf{T}_{00}=\frac{1}{2}\left(\left|\partial_{t} \phi\right|^{2}+\left|\partial_{x} \phi\right|^{2}\right)$ we conclude that all first derivatives of the map $\phi$ are bounded. Therefore, in $\mathbf{M}^{1+1}$, all wave maps, which are initially smooth remain so.

The proof we have presented is typical to the sweeping simplifications which occur only in $1+1$ dimensions. The case of wave maps defined in $\mathbf{M}^{1+2}$ is already much more complicated. We can proceed as before and classify the (W.M.) according to the scale associated to the total energy $E$. Thus, prescribing to the space-time variables the scale $L$ and to $\phi$ the scale $L^{0}$ we find that $\mathrm{E}$ has the scale $L^{s}$ with $s=n-2$. Consequently the (W.M.) is subcritical in $\mathbf{M}^{1+1}$, critical in $\mathbf{M}^{1+2}$ and supercritical in $\mathbf{M}^{1+n}, n \geq 3$. Under reasonable geometric assumptions we expect global regularity in the critical case $\mathbf{M}^{1+2}$. This conjecture has been recently checked for wave maps satisfying additional 
symmetry assumptions, see [Ch-Za] in the case of spherical symmetry and [Sh-Za], [Gr2] in the equivariant case.

Of course, we also know examples of partial differential equations, for which blow-up actually occurs. This is the case of the Burger equation

$$
u_{\ell}+u u_{x}=0 .
$$

Despite the fact that the equation has, not only one but infinitely many positive conserved quantities, e.g $\int|u(t, x)|^{2 k} d x$, any initial condition $u_{0}(x) \in C_{0}^{\infty}(\Re)$ leads to blow-up in finite time ${ }^{3}$ Remark that the Burger equation is supercritical relative to the total energy $E=\left(\int|u(t, x)|^{2} d x\right)^{1 / 2}$ and critical relative to the $L^{\infty}$ norm of $u$. Indeed if we prescribe to $t, x$ the same scale $L$ and to $u$ the scale $L^{0}$ then the conserved quantities $\left(\int|u(t, x)|^{2 k} d x\right)^{1 / 2 k}$ acquire the scales $L^{\frac{1}{2 k}}$

There are also known examples ${ }^{4}$ of finite time break-down of solutions for wavemaps in the supercritical case $\mathbf{M}^{3+1}$. But more important, from a physical point of view, is the well known fact that the Einstein equations also leads to singularities. It is interesting to remark in this respect that, relative to the total ADM mass, the Einstein field equations are supercritical ${ }^{5}$.

According to the classification we have indicated above it is widely expected that in subcritical situations break-down can be ruled out. We also believe that the same holds true in most critical problems. Finally, in the supercritical cases we have a lot of evidence that break-down can in fact occur.

Though we do not expect any bad behaviour in the subcritical problems, proving that it is indeed so is not necessarily an easy task. This is the case of the beautiful result of Eardley and Moncrief [E-M] for the Yang-Mills equations ${ }^{6}$ in $\mathbf{M}^{3+1}$. The proof required an insightful observation concerning the structure of the nonlinear terms of the Yang-Mills equations expressed in the Cronstrom gauge. As we have indicated above the question of regularity in the critical case has been solved for (N.W.E.) and is now the focus of considerable attention for (W.M.).

It is helpful to divide the general question of break-down and regularity into a sequence of simpler ones for which is easier to envision ongoing progress. Once more I will refer directly to the Einstein field equations and look at the other field theories from the perspective of (2a). The simplest of them all is,

Question 1. Under what assumptions on the initial conditions is the Cauchy problem locally well posed?

Technically this is the question of local in time existence and uniqueness of the development of an initial data set. Our present technology, based on energy estimates and Sobolev inequalities, requires too much differentiability on the initial data set.

3

4 see $[\mathrm{Sh}]$

5 Proceeding as before for (N.W.E.) and (W.M.) we assign to the space-time metric the scale $L^{0}$ and remark that the ADM mass $E=\frac{1}{16 \pi} \lim _{r \rightarrow \infty} \int_{S_{r}} \sum_{i, j}\left(\partial_{i} g_{i j}-\partial_{j} g_{i i}\right) N^{j} d a$ has the "supercritical" scale $L^{1}$.

6 Proceeding as before we associate to the vector potential $A$ the scale $L^{-1}$ and to the electromagnetic field $F$ the scale $L^{-2}$. Thus the total encrgy at time $t$ has the scale $L^{n-1}$ which is subcritical for $n-3$. 
Lowering these differentiability requirements is crucial in understanding the regularity properties of the solutions to $(\mathrm{E}-\mathrm{V})$ and also the general Einstein equations in the presence of matter.

Question 2. Under what assumptions on the initial data sets do there exist global, smooth and geodesically complete solutions of the Einstein-Vacuum equations?

This question is intimately connected to that of stability of the Minkowski spacetime. Namely the Minkowsky space-time $\mathbf{M}^{3+1}$ is a special solution of $\mathrm{E}-\mathrm{V}$ free of singularities. As mentioned above an initial data set is said to be flat if its development is diffeomorphic to $\mathbf{M}^{3+1}$. It is thus natural to ask what happens to the developments of initial data sets which are small perturbations of a flat initial data set. The answer to this question is not only important in view of the general program of studying the regularity of solutions evolving from regular Cauchy data but also in regard to the question of the structure of null infinity, of general solutions which evolve from asymptotically flat initial data sets. Indeed any A.F. initial data set can be interpreted, outside a sufficiently large relatively compact set $\mathcal{K}$, as a small perturbation of a flat initial data set. Thus the methods used in the study of the global stability of the Minkowski space-time can also be used in the study of the asymptotic properties of the development of any A.F. initial data set outside the future set of a sufficiently large set $\mathcal{K} \subset \mathcal{H}$.

The problem of the stability of the Minkowski space-time has been recently addressed in my joint work with D. Christodoulou [Ch-K12]. The result which we were able to prove asserts the following,

Theorem(CH-Kl). Any S.A.F. ${ }^{7}$ initial data set which satisfies, in addition, a Global Smallness Assumption, leads to a unique, smooth and geodesically complete development, solution of the Einstein-Vacuum Equations. Moreover, this development is globally asymptotically flat, by which we mean that its Riemann curvature tensor approaches zero ${ }^{8}$ on any causal or space-like geodesic, as the corresponding affine parameter tends to infinity.

The global smallness assumption requires that an appropiate $L^{2}$-norm of up to 2 derivatives of the curvature tensor of $\mathrm{g}$ and 3 derivatives of $\mathrm{k}$ are small. A more primitive version of our result requires one derivative less, in line with the statement of the local existence theorem. Further improvements of our result will depend crucially on progress made on Question 1.

Question 3. Under what conditions do initial data sets develop black holes and singularities?

The famous incompleteness theorem of Penrose asserts that if an initial data set of a space-time verifying the Einstein field equations (with very general assumptions on the energy-momentum tensor $T$ ) has a trapped sphere ${ }^{9}$ then some outgoing null geodesics normal to $S$ must be future-incomplete. Though the Penrose theorem indicates that

7 of order $k=4$. We note that the precise fall-off conditions of the initial data set are in fact given in $L^{2}$-weighted norms, and thus differ slightly from those we have discussed below

8 Our result gives precise information on the rate of decay of different components of the curvature tensor.

${ }^{9}$ i.e. a space-like sphere $S$ on $\mathcal{H}$ with a compact filling such that the outgoing null normal to $S$ are everywhere converging 
singularities can indeed occur for general solutions of the Einstein equations it remains entirely unclear what is the nature of these singularities and, more important, if the trapped sphere hypothesis is of any relevance in the actual process of a gravitational collapse.

At the present time we have no results concerning the formation of black holes for the $(\mathrm{E}-\mathrm{V})$ equations. Since the general problem is too hard one needs to look first at simplified situations. Unfortunately the (E-V) does not allow interesting A.F. solutions with additional symmetries, e.g. spherically symmetric. To overcome this one has to consider the general Einstein field equations coupled with some matterfield. The simplest such field is the linear scalar wave equation (S.W) with $V=0$. This coupling allows one to obtain nontrivial dynamics even in the spherically symmetric case. In this case the group $S O(3)$ acts as an isommetry group on the space-time $(\mathcal{M}, \mathbf{g})$. The group orbits are space-like metric 2-spheres $S$ of Gauss curvature $r^{-2}$ where $r$ is the area radius of $S$, i.e. $A(S)=4 \pi r^{2}$. Due to the $S O(3)$ symmetry the field equations can be reduced to quotient $Q$ of the space-time by the group. This a 2-dimensional manifold with boundary. The boundary corresponds to the set of fixed points of the group action and forms a time-like geodesic $\Gamma$. Choosing, on $Q$, a pair of conjugate optical functions $u, v$ with $u$ constant on any future directed null curve initiating at $\Gamma, v$ constant along each of the conjugate familly of null curves and such that both functions are increasing towards the future, the induced metric on $Q$ has the form $-\Omega^{2} d u d v$. Remark that $u, v$ are determined up to general transformations of the form $u \mapsto f(u), v \mapsto f(v)$ with $\mathrm{f}, \mathrm{g}$ arbitrary increasing functions. The reduced field equations form a system on $Q$ for the functions $r, \Omega, \phi$,

$$
\begin{aligned}
r \frac{\partial^{2} r}{\partial u \partial v} & =-\frac{1}{4} \Omega^{2}-\frac{\partial r}{\partial u} \frac{\partial r}{\partial v} \\
\frac{\partial^{2} r}{\partial u^{2}} & =2 \Omega^{-1} \frac{\partial r}{\partial u} \frac{\partial \Omega}{\partial u}-r\left(\frac{\partial \phi}{\partial u}\right)^{2} \\
\frac{\partial^{2} r}{\partial v^{2}} & =2 \Omega^{-1} \frac{\partial r}{\partial v} \frac{\partial \Omega}{\partial v}-r\left(\frac{\partial \phi}{\partial v}\right)^{2} \\
r \frac{\partial^{2} \phi}{\partial u \partial v} & =-\frac{\partial r}{\partial u} \frac{\partial \phi}{\partial v}-\frac{\partial r}{\partial v} \frac{\partial \phi}{\partial u}
\end{aligned}
$$

The above system is invariant under the transformations $u \mapsto f(u), v \mapsto g(v)$ and $\Omega \mapsto\left(f^{\prime} g^{\prime}\right)^{1 / 2} \Omega$.

The program of studying the spherically symmetric solutions of the coupled Einsteinscalar wave equation was initiated and carried out with remarkable success by D. Christodoulou, see [Ch2],[Ch3]. A basic ingredient of his analysis relies on the monotonicity properties of the mass function $m$ defined by the formula $1-\frac{2 m}{r}=-4 \Omega^{-2} \frac{\partial r}{\partial u} \frac{\partial r}{\partial v}$. We have $\frac{\partial m}{\partial v} \geq 0$ and $\frac{\partial m}{\partial u} \leq 0,=0, \geq 0$ according to whether $1-\frac{2 m}{r} \leq 0,=0, \geq 0$. In [Ch2] Christodoulou combines this montonicity properties of $m$ together with the powerful method of characteristics, typical to 2-dimensional hyperbolic problems, to prove a deep result concerning the formation of black holes for the system (E-S.W.). $\mathrm{He}$ considers the evolution of regular initial conditions defined on an outgoing null curve $C_{0}^{+}$, starting at a point on $\Gamma$, and shows that under reasonable assumptions of the data trapped spheres must form.

Question 4. What are the global regularity features of arbitrary devlopments of A.F. initial data sets? 
One of the main distant goals of the mathematical theory of General Relativity is to give a correct formulation and solve what is referred to as the "Cosmic Censorship" conjecture. Loosely speaking the conjecture asserts that there are no singularities outside black holes or, more picturesque, there exist no naked singularities.

Once again the understanding of the global regularity properties of the general Einstein equations is beyond our reach. To make progress we have to either restrict our attention to solutions with spherical symmetry or to consider simpler field theories. Concerning the first approach I have to mention once more the pioneering work of Christodoulou on the coupled E-S.W. equations. Following his result on the formation of black holes, which was mentioned above, he has concentrated his efforts on the regularity of solutions outside black holes. His results show that though "naked singularities" are possible they are unstable in the sense that the initial conditions from which these could evolve form an exceptional set of strictly positive codimension in the set of all allowable initial conditions.

In what follows I will give a short description on some of the progress made recently on the questions 1,3,4 and give a short description of the proof of Theorem ( $\mathrm{Ch}-\mathrm{Kl}$ ) mentioned in connection with question 2.

Q1. As we have mentioned below the first solution to this problem was given by Y.C. Bruhat, see [Br1], under the assumption that the initial metric $g$ has 3 derivatives, and the tensor $k$ has 2 derivatives locally on $L^{2}(\mathcal{H})$. Her proof is based on two ingredients. The first consists on an auxilliary construction of wave coordinates relative to which the $E-V$ equations take the form of a system of nonlinear wave equations. The special choice of wave coordinates is in fact not important, they can be replaced by other more geometric structures ${ }^{10}$. The second ingredient is more basic as it reflects on our present, limited, techniques in dealing with the local theory for all systems of nonlinear hyperbolic equations in more than 2 space-time dimensions ${ }^{11}$. It combines standard energy estimates for derivatives of solutions of the hyperbolic system together with Sobolev inequalities and an iteration procedure. The procedure has a built in limitation, as it requires too many derivatives on the initial data. For $E-V$ it means that we have to restrict ourselves to initial data sets with one derivative of the curvature tensor and two derivatives of $k$ in $L_{l o c}^{2}(\mathcal{H})$. This limitation is very significant when one passes from the local to the global study of the regularity properties of the system. To overcome the limitation it seems imperative to develop new analytic techniques. It helps, of course, to first address this question for a much simpler field theory. A good example is provided by the equations of Wave Maps from the Minkowski spacetime $\mathbf{M}^{n+1}$ to an arbitrary Riemannian manifold $(\mathcal{N}, h)$. Recall that, relative to

10 In [Ch-K12] the local existence theorem is proved relative to a maximal foliation. In other words the space-time is constructed locally together with a "time function" $t$ whose level hypersurfaces are maximal, i.e. $\operatorname{tr} k=0$.

11 In 2 space-time dimensions the situation is radically different because of the method of characteristics which allows better results based on the method of characteristics. This romains true in higler dimensions if ane considers only solutions with spherical symetry. 
a local chart in $\mathcal{N}$, the W.M. equations take the form,

$$
\square \phi^{I}+\sum_{J, K^{K}} \Gamma_{J K^{K}}^{I}(\phi) Q\left(\mathbf{D} \phi^{J}, \mathbf{D} \phi^{K}\right), \quad I=1, \ldots, N
$$

where,

$$
Q(\mathbf{D} \phi, \mathbf{D} \psi)=\mathbf{D}_{\alpha} \phi \mathbf{D}^{\alpha} \psi=\mathbf{m}^{\alpha \beta} \mathbf{D}_{\alpha} \phi \mathbf{D}_{\beta} \psi
$$

Here $\phi=\left(\phi^{I}\right)_{I=1, \ldots, N}$ is a vector valued function defined on $\mathbf{M}^{3+1}$. The $\Gamma_{J K}^{I}$ 's are given arbitrary smooth functions of $\phi$.

We consider the initial value problem on the hyperplane $t=x^{0}=0$,

$$
\phi(0, x)=f_{0}(x), \quad \partial_{t}(0, x)=f_{1}(x) .
$$

The classical local existence result for equations of type (1) requires $f_{0} \in H_{3}\left(\mathbf{R}^{3}\right)$, $f_{1} \in H_{2}\left(\mathbf{R}^{3}\right)$. Recently, in collaboration with $\mathrm{M}$. Machedon [Kl-Ma] we were able to prove the following,

Theorem (Kl-Ma). Consider systems of nonlinear wave equations in $\mathrm{M}^{3+1}$ of the type (1), (2) subject to the initial conditions (3) under the assumptions $f_{0} \in H_{2}\left(\mathbf{R}^{3}\right)$, $f_{1} \in H_{1}\left(\mathbf{R}^{3}\right)$. There exists a $T_{*}>0$ and a unique solution $\phi$ defined in the slab $\mathcal{D}_{*}=\left[0, T_{*}\right] \times \mathbf{R}^{3}$ and verifying the following,

(i) $\sup _{\mathcal{D}_{*}}|\phi(t, x)|<\infty$

(ii) $\iint_{\mathcal{D}_{\text {. }}}\left(|Q(\phi, \phi)|^{2}+|\mathbf{D} Q(\phi, \phi)|^{2}\right) d t d x<\infty$

(iii) $\sup _{\left[0, T_{*}\right]}\|\phi(t, \cdot)\|_{H^{2}\left(\mathbf{R}^{3}\right)}<\infty$.

As we have mentioned above the $L^{2}$ estimates, provided by the energy method, are not enough to prove a result like this. On the other hand any attempt to replace $L^{2}$ with any other $L^{p}$ is known to fail (see [L]) even for the linear problem, in $\mathbf{M}^{n+1}$,

$$
\square \phi=F ; \phi(0, x)=f_{0}(x), \partial_{t}(0, x)=f_{1}(x) .
$$

The only other possibility to get additional information is to consider space-time integrals. The best known result of this type, for the wave equation (4), is due to Strichartz $[\operatorname{Str} 1,2]$ who has proved the following inequality,

$$
\|\phi\|_{L^{q}} \leq C\left(\left\|f_{0}\right\|_{H^{1 / 2}}+\left\|f_{1}\right\|_{H^{-1 / 2}}+\|F\|_{L^{q}}\right)
$$

where $L^{p}, L^{q}$ are space-time norms with exponents, $p=2 \frac{n+1}{n+3}, q=2 \frac{n+1}{n-1}$.

The novelty of our result consists of estimating, in space-time, the Lorentz invariant bilinear form $Q(\phi, \psi)$ and clerive for it an estimate which is stronger than that which could be derived from the Strichartz inequality. This allows us to gain a full derivative over the classical result. The basic new tool is contained in the following

Proposition. Let $\phi, \psi$ be solutions in $\mathbf{M}^{3+1}$ to the equations $\square \phi=F, \quad \square \psi=G$ with initial conditions $\phi(0, x)=f_{0}(x), \partial_{t} \phi(0, x)=f_{1}(x)$ and $\psi(0, x)=g_{0}(x)$, $\partial_{t} \psi(0, x)=g_{1}(x)$. Assume that $f_{0}, g_{0} \in H_{2}\left(\mathbf{R}^{3}\right), f_{1}, g_{1} \in H_{2}\left(\mathbf{R}^{3}\right)$ and that the integrals $\int_{0}^{T}\|\mathbf{D} F(t, \cdot)\|_{L^{2}\left(\mathbf{R}^{3}\right)} d t, \int_{0}^{T}\|\mathbf{D} F(t, \cdot)\|_{L^{2}\left(\mathbf{R}^{3}\right)} d t$ are both finite. Then the 
space-time integral $\int_{0}^{T} \int_{\mathbf{R}^{3}} \mid \mathbf{D} Q(\phi, \psi)^{2} d t d x$ is also bounded, with a bound depending only on the product of $\left(\left\|f_{0}\right\|_{H^{2}\left(\mathbf{R}^{3}\right)}+\left\|f_{1}\right\|_{H^{1}\left(\mathbf{R}^{3}\right)}+\int_{0}^{T}\|\mathbf{D} F(t, \cdot)\|_{L^{2}\left(\mathbf{R}^{3}\right)} d t\right)$ with $\left(\left\|g_{0}\right\|_{H^{2}\left(\mathbf{R}^{3}\right)}+\left\|g_{1}\right\|_{H^{1}\left(\mathbf{R}^{3}\right)}+\int_{0}^{T}\|\mathbf{D} G(t, \cdot)\|_{L^{2}\left(\mathbf{R}^{3}\right)} d t\right)$.

Remark 1. The result of the proposition remains true if we replace the quadratic form $Q$ by $Q_{\alpha \beta}(\phi, \psi)=\partial_{\alpha} \phi \partial_{\beta} \psi-\partial_{\alpha} \psi \partial_{\beta} \phi$. On the other hand the result is wrong if we replace the bilinear form $Q$ with any other bilinear form in the space-time gradients of $\phi, \psi$. The result of Theorem (Kl-Ma) remains true if we also allow the forms $Q_{\alpha \beta}$ but we believe is wrong if one considers in (1) general expressions quadratic in the $\mathbf{D} \phi$. This suggests that any future improvements of the classical local existence and uniqueness results require special features of the equations one is interested in.

Remark 2. The proof of Theoren (KI-Ma) depends heavily on the fact that the nonlinear terms in (1), (2) do not depend on the top derivatives. It is reasonable to believe however that the result of the theorem can be extended to certain classes of quasilinear equations. Most important we believe that a similar result holds true for the Einstein vacuum equations, in other words the (E-V) equations should be well posed for initial data sets $(\mathcal{H}, g, k)$ for which the components curvature tensor $R(g)$ and the first derivatives of $k$ are bounded in $L^{2}$.

Q2. In proving the result on the stability of the Minkowski space-time mentioned above we had to overcome the following major obstacles,

a. The problem of coordinates.

b. The strongly nonlinear character of the equations.

c. The long range character of the initial data set.

d. The nontrivial character of the asymptotic properties of the causal structure of any small perturbation of the Minkowski space-time.

e. The conjunction of all of the above.

The scope of this lecture doesn't allow me to discuss in detail each of the above difficulties and describe our strategy for overcoming them. For those interested I would like to refer to the extensive introduction of [Ch-Kl2] or the recent Bourbaki seminar [Bour]. In what follows I will only give a very short description of the main ideas in [Ch-Kl2].

The difficulty (a) is typical to General Relativity. In short one is faced with the following dilemma. To write the equations in a meaningful way one seems forced to introduce an additional structure, e.g. wave coordinates. Such a structure seems necessary even to allow the formulation of well posed Cauchy problem and a proof of a local in time existence result. Nevertheless, as the particular case of wave coordinates illustrates, the structure, if not carefully chosen, may lead, in the large, to problems of its own making. Indeeed, as pointed by $\mathrm{Y}$. Chocquet-Bruhat [Br2], the "wave coordinates" are unstable in the large even when one starts with initial conditions close to flat. In [Ch-Kl2] we solve this problem by constructing our space-times together with two additional geometric stuctures. One is given by a time function $t$ whose level hypersurfaces are maximal. The second, much more important, is given by the level hypersurfaces of an optical function. This is a special solution $u$ of the Eikonal equation $\mathrm{g}^{\alpha \beta} \partial_{\alpha} u \partial_{\beta} u=0$, whose level hypersurfaces are outgoing null hypersurfaces with correct asymptotic properties at null future infinity.

The other major obstacle in the study of the Einstein equations consists in their hyperbolic and strongly nonlinear character. As we have already mentioned the only 
apriori bounds we have available in the study of quasilinear hyperbolic equations, in the physical space-time dimension, are those based on energy estimates. Yet the classical energy estimates are limited to proving estimates which are local in time. The difficulty has to do with the fact that, in order to control the higher energy norms of the solutions, one has to control the integral in time of their bounds in uniform norm. For this we need to control the decay of the $L^{\infty}$ norms for which we have no direct information. This difficulty was overcome by us using a strategy based on two important ideas. The first has to do with the existence of the so called Bell-Robinson tensor which plays, for the Einstein field equations, a role simmilar to that played by the the cnergy-momentum tensor of the standard field theories of matter. The second is a technique, developed by us, of deriving all the asymptotic behaviour of a field based entirely on energy estimates. Recall that, if $\mathbf{T}$ is the energy-momentum tensor of a field theory we have,

$$
\mathbf{D}^{\nu} \mathbf{T}_{\mu \nu}=0 .
$$

Let $\mathrm{X}$ be an arbitrary vectorficld and $P$ be the 1 -form obtained by contracting the energy-momentum tensor with $X$ i.e. $P_{c \gamma}=\mathbf{T}_{\alpha \beta} X^{\beta}$. Then, since $\mathbf{T}$ is symmetric and divergencc-less,

$$
D^{\alpha} P_{\alpha}=\frac{1}{2} \mathbf{T}^{\alpha \beta} \pi_{\alpha \beta}
$$

where,

$$
\pi_{\alpha \beta}=D_{\beta} X_{\alpha}+D_{\alpha} X_{\beta}
$$

is the deformation tensor of $X$. We now integrate (6) on a lens shaped domain $\mathcal{D}$ be bounded by two space-like hypersurfaces $\mathcal{H}_{0}, \mathcal{H}_{1}$. In view of the divergence theorem we derive the integral identity,

$$
\int_{\mathcal{H}_{0}} \mathbf{T}(X, T) d a_{g}-\int_{\mathcal{H}_{1}} \mathbf{T}(X, T) d a_{g}=-\int_{\mathcal{D}} \mathbf{T}^{\alpha \beta} \pi_{\alpha \beta}
$$

where $T$ is the future oriented unit normal, $\mathrm{g}$ the induced metric and $d a_{g}$ the area element of $\partial \mathcal{D}$.

In the particular case when $X$ is Killing ${ }^{12}$ its deformation tensor $\pi$ vanishes identically and we derive the conservation law,

$$
\int_{\mathcal{H}_{0}} \mathbf{T}(X, T) d a_{g}=\int_{\mathcal{H}_{1}} \mathbf{T}(X, T) d a_{g}
$$

Remark that the result (9) remains true if $X$ is a conformal Killing vector field, i.e. it generates conformal isometries, and $\mathbf{T}$ is traceless. Indeed, if $X$ is conformal Killing, $\pi_{\alpha \beta}=\Lambda \mathbf{g}_{\alpha \beta}$ and hence $\pi_{\alpha \beta} \mathbf{T}^{\alpha \beta}=\Lambda \operatorname{tr}(\mathbf{T})=0$. One can easily show that if the action integral $\mathcal{S}[\psi, \mathbf{g}]$ is invariant under conformal rescalings of the metric, i.e. $\tilde{\mathbf{g}}=\Omega^{2} \mathbf{g}$, then the corresponding energy-momentum tensor is traceless. This is the case of the Yang-Mills Theory in $3+1$ dimensions.

The identities (8) and (9) are usually applied to time-like future oriented vectorfields $X$, in which case, in view of the positive energy condition, the integrand $\mathbf{T}(X, T)$ is positive. This is the essence of the standard energy method. The method allows us to obtain apriori bounds, typically $L^{2}$ on space-like hypersurfaces, of the field under 
consideration. Our main new idea is based on a simple extension of this method in situations when the background has symmetries or, more appropiate for the stability of the Minkowski space-time, approximate symmetries.

To illustrate the method consider the Maxwell equations in $\mathbf{M}^{3+1}$. As mentioned above the Maxwell theory is conformal invariant. This means that whenever $F$ is a solution and $X$ is an arbitrary conformal Killing vectorfield the Lie derivative of $F$ with respect to $\mathrm{X}$ is also a solution to the Maxwell equations. Combining this fact with the standard energy method described above we can now derive $L^{2}$ estimates for various vectorfields applied to $F$. For example, consider the following quantities,

$$
\begin{aligned}
\mathbf{Q}_{0}(t)= & \int_{\mathcal{H}_{t}} \mathbf{T}(F)\left(K_{0}, T_{0}\right) d x \\
\mathbf{Q}_{1}(t)= & \sum_{a=1}^{3} \int_{\mathcal{H}_{\imath}} \mathbf{T}\left(\mathcal{L}_{\Omega(a)} F\right)\left(\overline{K_{0}}, T_{0}\right) d x+\int_{\mathcal{H}_{t}} \mathbf{T}\left(\mathcal{L}_{S} F\right)\left(\overline{K_{0}}, T_{0}\right) d x \\
& +\int_{\mathcal{H}_{t}} \mathbf{T}\left(\mathcal{L}_{T_{0}} F\right)\left(\overline{K_{0}}, T_{0}\right) d x \\
\mathbf{Q}_{2}(t)= & \sum_{a, b=1}^{3} \int_{\mathcal{H}_{t}} \mathbf{T}\left(\mathcal{L}_{\Omega(a)} \mathcal{L}_{\Omega(b)} F\right)\left(\overline{K_{0}}, T_{0}\right) d x+\sum_{a=1}^{3} \int_{\mathcal{H}_{\imath}} \mathbf{T}\left(\mathcal{L}_{S} \mathcal{L}_{\Omega(a)} F\right)\left(\overline{K_{0}}, T_{0}\right) d x \\
& +\int_{\mathcal{H}_{t}} \mathbf{T}\left(\mathcal{L}_{S} \mathcal{L}_{S} F\right)\left(\overline{K_{0}}, T_{0}\right) d x+\int_{\mathcal{H}_{t}} \mathbf{T}\left(\mathcal{L}_{T_{0}} \mathcal{L}_{T_{0}} F\right)\left(\overline{K_{0}}, T_{0}\right) d x .
\end{aligned}
$$

Here $\mathcal{H}_{t}$ are the level hypersurfaces of the time function $t=x^{0}$ with future directed unit normal $T_{0}=\partial_{0} . T_{0}$ is also the generator of time translations. The vectorfields $\Omega(a)=\epsilon_{a b c} x_{b} \partial_{c}, a=1,2,3$ are the usual angular momentum operators. $S=x^{\alpha} \partial_{\alpha}$ is the generator of dilations while $\bar{K}_{0}=K_{0}+T_{0}$ with $K_{0}=\left(t^{2}+r^{2}\right) \partial_{0}+2 t x^{i} \partial_{i}$ the generator of inverted time translations.

In view of the above discussion the quantities $\mathbf{Q}_{0}(t), \mathbf{Q}_{1}(t), \mathbf{Q}_{2}(t)$ are all time independent and therefore bounded for all $t \in \Re$ if $\mathbf{Q}_{i}(0), i=0,1,2$ are all bounded. These $L^{2}$ bounds can be combine with some global version of the classical Sobolev inequalities to derive very sharp uniform estimates for $F$.

To do this we decompose $F$ relative to the pair of conjugate null vectors $e_{+}=$ $\partial_{t}+\sum_{i=1}^{3} \frac{x^{i}}{r} \partial_{i}, e_{-}=\partial_{t}-\sum_{i=1}^{3} \frac{x^{i}}{r} \partial_{i}$ and an arbitrary orthonormal frame $\left(e_{A}\right)_{A=1,2}$ on the 2-spheres $S_{u, v}$ obtain by the intersection of the level hypersurfaces of $u=t-r$ with those of $v=t+r$, into the vectors $\alpha_{A}=F\left(e_{+}, e_{A}\right), \underline{\alpha}_{A}=F\left(e_{-}, e_{A}\right)$ and scalars $\rho=\frac{1}{2} F\left(e_{+}, e_{-}\right) \sigma=\frac{1}{2}^{*} F\left(e_{+}, e_{-}\right)$. This is called the null decomposition of $F$. We can now state the following,

Proposition 1 [Ch-Kl1]. Let $F$ be a solution of $(M)$ and assume that the initial conditions on the hyperplane $t=0$ are such that the quantities $\mathbf{Q}_{i}(0), i=0,1,2$ are all bounded. Consider the asymptotic behaviour of $F$ for $t>0$. In the space-time region $r \leq 1+\frac{t}{2}$ all components of $F$ behave in the same way, $|F(t, x)| \leq c(1+t)^{-\frac{5}{2}}$. Outside that region ${ }^{13}$ we have, $|\alpha(t, x)| \leq(1+t+r)^{-\frac{6}{2}},|\rho(t, x), \sigma(t, x)| \leq(1+t+r)^{-2}(1+\mid t-$ $r \mid)^{-\frac{1}{2}},|\underline{\alpha}(t, x)| \leq(1+t+r)^{-1}(1+|t-r|)^{-\frac{3}{2}}$.

13 The crucial region, where different null components of $F$ decay in different ways, is the wave zone region where $u=t-r$ is bounded. 
We have thus been able to derive the asymptotic properties of the Maxwell-equations relying only on their geometric properties. This feature is crucial in applications to nonlinear problems. A similar method lies also at the heart of our proof of the stability of the Minkowski space-time. It consists in the derivation of $L^{2}$ estimates for the modified Lie derivatives of the curvature tensor $\mathbf{R}$ of a space-time $\mathcal{M}$, $\mathbf{g}$ verifying the Einstein-Vacuum equtions ( $\mathrm{E}-\mathrm{V}$ ) relative to some modification of the vectorfields $T_{0}, \Omega, S, \overline{K_{0}}$, as above. This is based on the crucial observation that the Bianchi identities,

$$
\mathbf{D}_{\epsilon} \mathbf{R}_{\alpha \beta \gamma \delta}+\mathbf{D}_{\alpha} \mathbf{R}_{\beta \epsilon \gamma \delta}+\mathbf{D}_{\beta} \mathbf{R}_{\epsilon \alpha \gamma \delta}=0
$$

if interpreted as equations satisfied by $\mathbf{R}$ relative to a fixed background metric $\mathbf{g}$, are conformally covariant. The role of the Bell-Robinson tensor, $\mathbf{T}_{\alpha \beta \gamma \delta}=$ $\frac{1}{2}\left(\mathbf{R}_{\alpha \mu \beta \nu} \mathbf{R}_{\gamma}{ }^{\mu}{ }_{\delta}{ }^{\nu}+{ }^{\star} \mathbf{R}_{\alpha \mu \beta \nu}{ }^{*} \mathbf{R}_{\gamma}{ }^{\mu}{ }_{\delta}{ }^{\nu}\right)$ where, ${ }^{\star} \mathbf{R}_{\alpha \beta \gamma \delta}=\frac{1}{2} \in_{\alpha \beta \mu \nu} \mathbf{R}^{\mu \nu}{ }_{\gamma \delta}$, plays the same role for $(\mathrm{B})$ as that played by the energy-momentum tensor before. Indeed $\mathbf{T}$ exhibits all the propertics of an energy-momentum tensor. Thus ${ }^{14} \mathbf{T}$ is symmetric relative to all pair of indices and satisfies the conservation law, $\mathbf{D}^{\delta} \mathbf{T}_{\alpha \beta \gamma \delta}=0$. Moreover it verifies the positive energy condition, i.e. $\mathbf{T}(X, Y, X, Y)$ is positive whenever $X, Y$ are future directed time-like vectors. The conformal covariance of (B) translates into the very important fact that $\mathbf{T}$ is traceless. Also, if we define the modified Lie clerivative of $\mathbf{R}$ relative to a vectorfield $\mathrm{X}$ by the formula, $\hat{\mathbf{L}}_{X} \mathbf{R}=\mathbf{L}_{X} \mathbf{R}_{\alpha \beta \gamma \delta}-\frac{1}{2}\left(\pi_{\alpha}^{\mu} \mathbf{R}_{\mu \beta \gamma \delta}+\pi_{\beta}^{\mu} \mathbf{R}_{\alpha \mu \gamma \delta}+\pi_{\gamma}^{\mu} \mathbf{R}_{\alpha \beta \mu \delta}+\pi_{\delta}^{\mu} \mathbf{R}_{\alpha \beta \gamma \mu}\right)+\frac{3}{8} \operatorname{tr} \pi \mathbf{R}_{\alpha \beta \gamma \delta}$, we see that the commutation of $\hat{\mathbf{L}}_{X}$ with (B) produces error terms depending on the vector $X$ only through the trace-less part of its deformation tensor $\pi$. Thus, if the background metric is that of the Minkowski space-time the modified Lie derivative relative to a conformal Killing vectorfield commutes with the Bianchi equations (B). These properties of (B) allow us to define generalized energy norms for $R$, as we have done previously for $F$. These norms allow us to control the asymptotic behaviour of $\mathbf{R}$. We illustrate this in Minkowski space-time as follows. Assume that $W$ is a 4-covariant trace-less tensor verifying all the symmetry properties of the curvature tensor $\mathbf{R}$. Assume that $W$ is a solution of the Bianchi equations (B) in $\mathbf{M}^{3+1}$. Let $\mathbf{Q}_{0}(t), \mathbf{Q}_{1}(t), \mathbf{Q}_{2}(t)$ be the quantities,

$$
\begin{aligned}
\mathbf{Q}_{0}(t)= & \int_{\mathcal{H}_{t}} \mathbf{T}(W)\left(\overline{\bar{K}_{0}}, \overline{\Gamma_{0}}, T_{0} T_{0}\right) d x \\
\mathbf{Q}_{1}(t)= & \sum_{a=1}^{3} \int_{\mathcal{H}_{t}} \mathbf{T}\left(\hat{\mathcal{L}}_{\Omega(a)} W\right)\left(\overline{K_{0}}, \overline{K_{0}}, T_{0} T_{0}\right) d x \\
& +\int_{\mathcal{H}_{t}} \mathbf{T}\left(\hat{\mathcal{L}}_{T_{0}} W\right)\left(\overline{K_{0}}, \overline{K_{0}}, \overline{K_{0}} T_{0}\right) d x \\
\mathbf{Q}_{2}(t)= & \sum_{a, b=1}^{3} \int_{\mathcal{H}_{t}} \mathbf{T}\left(\hat{\mathcal{L}}_{\Omega(a)} \hat{\mathcal{L}}_{\Omega(b)} W\right)\left(\overline{K_{0}}, \overline{K_{0}}, T_{0}, T_{0}\right) d x \\
& +\sum_{a=1}^{3} \int_{\mathcal{H}_{t}} \mathbf{T}\left(\hat{\mathcal{L}}_{\Omega(a)} \hat{\mathcal{L}}_{T_{0}} W\right)\left(\overline{\bar{K}_{0}}, \overline{K_{0}}, \overline{\Gamma_{0}}, T_{0}\right) d x
\end{aligned}
$$

14 These properties hold true as a consequence of the symmetries of $\mathbf{R}$ and, in view of the $\mathrm{E}-\mathrm{V}$ equations, the condition $\mathbf{R}_{\alpha \beta}=0$. 


$$
\begin{aligned}
& +\int_{\mathscr{H}_{i}} \mathrm{~T}\left(\hat{\mathcal{L}}_{S} \hat{\mathcal{L}}_{T_{0}} W\right)\left(\overline{K_{0}}, \overline{K_{0}}, \overline{K_{0}}, T_{0}\right) d x \\
& +\int_{\mathcal{H}_{i}} \mathrm{~T}\left(\hat{\mathcal{L}}_{T_{0}} \hat{\mathcal{L}}_{T_{0}} W\right)\left(\overline{K_{0}}, \overline{K_{0}}, \overline{K_{0}}, T_{0}\right) d x .
\end{aligned}
$$

with $T_{0}, \Omega, S, \overline{K_{0}}$ as in $(10)$.

The null decomposition of $W$ is given by $\alpha_{A}=W\left(e_{A}, e_{+}, e_{B}, e_{+}\right), \beta_{A}=$ $\frac{1}{2} W\left(e_{A}, e_{+}, e_{-}, e_{+}\right), \rho=\frac{1}{4} W\left(e_{-}, e_{+}, e_{-}, e_{+}\right), \sigma=\frac{1}{4}^{*} W\left(e_{-}, e_{+}, e_{-}, e_{+}\right), \underline{\beta} \underline{A}_{A}=$ $\frac{1}{2} W\left(e_{A}, e_{-}, e_{-}, e_{+}\right), \underline{\alpha}_{A B}=W\left(e_{A}, e_{-}, e_{B}, e_{-}\right)$. We can now state the following,

Proposition 2. Let $W$ be a solution of the Bianchi equations in $\mathbf{M}^{3+1}$ and assume that the initial conditions on the hyperplane $t=0$ are such that the quantities $\mathbf{Q}_{i}(0)$ are all bounded. Consider the asymptotic behaviour of $W$ for $t>0$. In the spacetime region $r \leq 1+\frac{t}{2}$ all components of $W$ behave in the same way, $|W(t, x)| \leq$ $c(1+t)^{-\frac{7}{2}}$. Outside that region we have, $|\alpha(t, x)| \leq(1+t+r)^{-\frac{7}{2}}, \beta \leq(1+t+r)^{-\frac{7}{2}}$, $|\rho(t, x), \sigma(t, x)| \leq(1+t+r)^{-3}(1+|t-r|)^{-\frac{1}{2}},|\underline{\beta}| \leq(1+t+r)^{-2}(1+|t-r|)^{-\frac{3}{2}}$ $|\underline{\alpha}(t, x)| \leq(1+t+r)^{-1}(1+|t-r|)^{-\frac{r}{2}}$.

Both Propositions 1,2 have been proved in [Ch-Kl1].

The implementation of a similar strategy for the actual $\mathrm{E}-\mathrm{V}$ equations requires some fundamental modifications. We rely on the same quantities ${ }^{15} \mathbf{Q}_{1}(t), \mathbf{Q}_{2}(t)$ introduced in (11). The major departure from the linear theory of Prop. 2 is that the vectorfields $T_{0}, \Omega, S, K_{0}$ are now themselves unknown and have to be constructed together with our space-time. This is due to the difficulty (d). Indeed, if the asymptotic behaviour of the causal structure of the space-time we construct would have been trivial we could have chosen as vectorfields $T_{0}, \Omega, S, K_{0}$ the ones given to us in $\mathbf{M}^{3+1}$. As it stands, due to the mass term which appears in the Schwarzschild part of an (S.A.F.) initial data set, has the long range effect of changing the asymptotic position of the null geodesic cones relative to the maximal foliation. They are expected to diverge logarithmically from their corresponding position in flat space-time. In addition to this their asymptotic shear ${ }^{16}$ differs drastically from that in the Minkowski spacetime. This difference reflects the presence of gravitational radiation in any nontrivial perturbation of the Minkowski space-time. To take this effect into account we rely heavily on our optical function $u$ and construct the vectorfields $T_{0}, \Omega, S, K_{0}$ based on its properties ${ }^{17}$. The construction of $u$ itself is very elaborate and requires a systematic study of the geometry of null hypersurfaces.

In linear theory the time derivatives of the $\mathbf{Q}_{1}, \mathbf{Q}_{2}$ are zero. In the case of the $(\mathrm{E}-\mathrm{V})$ equations they give rise to cubic error ${ }^{18}$ terms which depend linearly on the traceless part of the deformation tensors of $K_{0}, T, S, \Omega$, and quadratic with respect to $\mathbf{R}$ and its covariant and Lie derivatives in the direction of $T, S, \Omega$. The crucial point of our overall strategy is to control the time integral of these error terms. This depends on the one

15 Because of the difficulty (c) we have to avoid the use of $\mathbf{Q}_{0}(t)$ which would be infinite at $t=0$.

16 the traceless part of their null second fundamental form

17 and also those of the maximal foliation induced by the time function $t$.

18 gentrated each time we commute the Bianchi Identities with a one of the vertorfields used in the definition of $\mathbf{Q}_{1}, \mathbf{Q}_{2}$ 
hand on the asymptotic behaviour of all components of $\mathbf{R}$ and its covariant derivatives, which are themselves controlled by the basic quantities $\mathbf{Q}_{1}, \mathbf{Q}_{2}$. On the other hand it depends on the asymptotic behavior of the deformation tensors of our vectorfields, and finally, due to the general covariance of the equations, on the cancellations of the "worst possible" cubic terms. It is well known that arbitrary quadratic nonlinear perturbations of the scalar wave equation, even when derivable from a Lagrangean, could lead to formation of singularities unless a certain structural condition, which we have called the Null condition, is satisfied (see [K12], anad [Ch1], [Kl2]). It turns out that the appropriate, tensorial version of this structural condition is satisfied by the Einstein equations. Roughly speaking one could say that the troublesome nonlinear terms, which could have led to formation of singularities, are in fact excluded due to to the covariance and algebraic properties ${ }^{19}$ of the Einstein equations.

Putting together all the elements discussed above requires an elaborate boot-strap argument based on the method of continuity. In the end we show that $\mathbf{Q}_{1}, \mathbf{Q}_{2}$ can never become large, if they are sufficiently small at $t=0$. The local existence theorem then guarantees that we can extend our space-time indefinitely.

Finally it remains to note that our work has many important conclusions. Far from beeing an abstract proof of existence it provides precise information on the asymptotic nature of gravitational radiation. Some of our conclusions confirm the non-rigurous results ${ }^{20}$ obtained by Bondi, Sachs, Penrose etc.

Some of them are however new. One of these has lead D. Christodoulou to a real experimental prediction. In [Ch4] he shows that gravitational waves generated by astronomical sources can have a nonlinear effect on laser interferometer detectors on Earth. This effect is shown to be of the same order of magnitude as the linear effects upon which all previous efforts to detect gravitational waves were based. Moreover the nonlinear effect is shown to produce a permanent displacement of test masses after the passage of a wave. It can thus alter significantly the startegy upon which the experimentalists plan to build their future detectors.

19 These basic algebraic properties of the Einstein equations, which allow us to prove the above stated global existence result, are in sharp contrast with the nonlinear hyperbolic equations of classical continuum mechanics. Indeed the equation of Nonlinear Elasticity [John] and of Compressible fluids [Si], in four space and time dimensions, form singularities even for arbitrary small initial conditions.

20 I want to point out however that our results are inconsistent with the assumption, made by Penrose [Pe1], [Pe2], concerning the smoothness of null infinity. Our results show weaker "peeling" than those implied by the Penrose requirement. It remains questionable whether there are any smooth initial conditions which lead to Ricci flat space-times for which the Penrose requirement is valid. 


\section{References}

[Bour] J.P Bourguignon, "Stabilite par deformation non-lin. de la metrique de Minkowski," Seminaire N. Bourbaki 1900-1901 June 1991.

[Br1] C. Bruhat, "Theoreme d'existence pour certain systemes d'equations aux deriveès partielles nonlinaires," Acta Matematica 88, 1952, 141-225.

[Br2] C. Bruhat, "Un theoreme d'instabilite pour certain equations hyperb. non-lineaire," C.R. Acad. Sci. Paris 276A, 1973, pp. 281.

[Ch1] D. Christodoulou, "Global solutions for nonlinear hyperbolic equations for small data," Comm. Pure Appl. Math. 39 1986, 267-282.

[Ch2] D. Christodoulou, "A Mathematical Theory of Gravitational Collapse," Comm. Math. Ph. 108, p 613 (1987).

[Ch3] D. Christodoulou, "The Formation of Black Holes and Singularities in Spherically Symmetric Gravitational Collapse," CP. Appl. Math. 44, 339-373(1991).

[Ch4] D. Christodoulou, "The Nonlinear Nature of Gravitation and Gravitational Wave Experiments," Preprint June 1991.

[Ch-KI1] D. Christodoulou-S.Klainelman, "Asymptotic Properties of Linear Field Equations in Minkowski Space-Time," Comm. Pure Appll. Math. 43 137-199(1990).

[Ch-K12] D. Christodoulou-D. S.Klainel'man, "The nonlinear stability of the Minkowski space-time," To appear in Princeton Univ. Pless .

[Ch-Za] D. Christodoulou-A. Shadi Tahvildar-Zadeh, "Regularity of Spherically Smmetric Farmonic Maps of the $2+1$ dim. Minkowski Space," Preprint.

[E-M] D. Eardley-V. Moncricf, "The Global Existence of Yang-Mills-IIiggs fields in $\mathbf{M}^{3+1}$," C.M.P. 83, 171-212(1982).

[Gr1] M. Grillakis, "Regularity and Asympt. Behaviour of the Wave Eq. with as critical nonlinearity," Ann. of Math. 132, 485-509(1990).

[Gr2] M. Grillakis, "Classical solutions for the Equivariant Wave Maps in $1+2$ dimensions," preprint.

[Gu] Chao, Gu, Hoo, "On the Cauchy PR for harmonic maps defined on 2-D Minkowski spacetime," C.P.A.M. 33, 1980, pp. 727-737.

[Jö] K. Jörgens, "Das Angfangswertproblem im Grossen Fur eine Klasse nichtlinearer Wellengleichungen," Math. Z., 77, 295-307( 1961).

[John] F. John, "Formation of Singularities in Elastic Waves," Lecture Notes in Phys., Springer-Verlag, 1984, pp. 190-214.

[Kl1] S. Klainerman, "Uniform decay estimates and the Lorentz invariance of the classical wave equation," Comm. Pure. Appl. Math. 38, 1985, 321-332.

[K12] S. Klainerman, "Long time behaviour of solutions to nonlinear wave equations," Proc, of the I.C.M. Warsaw, 1982, pp. 1209-1215.

[Kl3] S. Klainerman, "The null condition and global existence to nonlinear wave equations," Lect. Appl. Math. 23, 1986, 293-326.

[Kl4] S. Klainerman, "Remarks on the global Sobolev inequalities in Minkowski space," Comm. Pure Appl. Math. 40, 1987, 111-117.

[Kl-Ma] S. Klainerman-M.Machedon, "Space-Time estimates for null forms and the local existence theorem," preprint.

[L] W. Littman "The wave operator and $L^{p}$ norms," Journal of Math. and Mech. 12 pp. 55-68(1963).

[Pe1] R. Penrose, Structure of Space-Time, Batelle Rencontre, C.M. DeWitt and J.A. Wheeler, 1967.

[Pe2] R. Penrose, "Zero rest mass fields including gravitation: asymptotic behaviour," Proc. Roy. Soc. Lond. A284, 1962, 159-203.

[Sh-Stru] work in progress.

[Sh] "Weak solutions and development of singularities for the SU(2) $\sigma$-model," Comm. P. Appl. Math. 1988, pp. 459-469.

[Sh-Za] J. Shatah, "A. Tahvildar-Zadeh Regularity of harmonic maps from the Minkowski spacetime into rotationally symmetric manifolds," preprint.

[Si] T. Sideris, "Formation of Singularities in 3-d Compressible Fluids," Comm. Math. Phys. $101,1985,275-485$. 
[Str1] R.S. Strichartz, "A Priori Estimates for the Wave Equation and some Applications," J. of Funct. Anal.5, 218-235(1970).

[Str1] R.S. Strichartz, "Restrictions of Fourier Transforms to Quadratic Surfaces and Decay of Solutions of Wave Eq." Duke Math. J. 44, No 3, 705-714 (1977).

[Stru] M. Struwe, "Globally Regular Solutions to the $\Phi^{5}$ - Klein-Gordon equation." 
